BULL. AUSTRAL. MATH. SOC.

VOL. $18(1978), 55-64$.

\title{
Oscillation theorems for semilinear hyperbolic and ultrahyperbolic operators
}

\section{Mamoru Narita}

The oscillation property of the semilinear hyperbolic or ultrahyperbolic operator $L$ defined by

$$
L[u] \equiv \Delta_{x} u-\sum_{i, j=1}^{m} \frac{\partial}{\partial y_{i}}\left\{a_{i j}(x, y) \frac{\partial u}{\partial y_{j}}\right)+f(x, y, u)
$$

is studied. Sufficient conditions are provided for all solutions of $u[u] \leq 0$ satisfying certain boundary conditions to be oscillatory. The basis of our results is the non-existence of positive solutions of the associated differential inequalities.

Oscillation criteria for linear hyperbolic differential equations have been obtained by Kahane [1], Kreith [2, 3], Pagan [7], Travis [8], and Young [9]. More recently, the author and Yoshida [5] established oscillation theorems for linear ultrahyperbolic operators. The purpose of this paper is to study the oscillation property of a class of nonlinear hyperbolic or ultrahyperbolic equations and inequalities. Use is made of some of the techniques and results developed by Naito and Yoshida [4] and Noussair and Swanson [6].

$$
\text { Let } x=\left(x_{1}, \ldots, x_{n}\right) \text { and } y=\left(y_{1}, \ldots, y_{m}\right) \text { denote points in } R^{n}
$$

and $R^{m}$, respectively. Let $H$ be an unbounded domain in $R^{n}$ defined by

Received 14 October 1977. The author would like to thank Professor T. Kusano for many helpful suggestions. 


$$
H=\left\{x=\left(x_{1}, \ldots, x_{n}\right): 0<x_{i}<\infty, i=1, \ldots, n\right\},
$$

and let $G$ be a bounded domain in $R^{m}$ with piecewise smooth boundary.

The partial differential operator to be considered in this paper is

$$
L[u] \equiv \Delta_{x} u-\sum_{i, j=1}^{m} \frac{\partial}{\partial y_{i}}\left(a_{i j}(x, y) \frac{\partial u}{\partial y_{j}}\right)+f(x, y, u),
$$

where $\Delta_{x}$ denotes the laplacian in $R^{n}$; that is, $\Delta_{x}=\frac{\partial^{2}}{\partial x_{1}^{2}}+\ldots+\frac{\partial^{2}}{\partial x_{n}^{2}}$.

The coefficients $a_{i j}(x, y)$ are real-valued functions of class $C^{1}(\overline{H \times G})$, $(i, j=1, \ldots, m)$, and $f(x, y, \xi)$ is a real-valued function of cless $C^{0}\left(\overline{H \times G} \times R^{1}\right)$. The matrix $\left(a_{i j}\right)$ is assumed to be symmetric and positive definite in $H \times G$. The domain $D_{L}$ of $L$ is the set of all real-valued functions of class $C^{2}(H \times G) \cap C^{1}(\overline{H \times G})$.

For each $u \in D_{L}$ we define the function $g(x)$ by

$$
g(x)=\frac{1}{\kappa} \int_{G} u(x, y) d y, \quad\left(\kappa=\int_{G} d y\right) .
$$

LEMMA 1. Assume that:

(i) $f(x, y, \xi) \geq p(x) \phi(\xi)$ for alz $(x, y) \in H \times G$ and for alz $\xi>0$, where $p$ is continuous and non-negative in $H$ and $\phi$ is continuous, non-negative, and convex in $(0, \infty)$;

(ii) $u(x, y) \in D_{L}$ is a positive solution of the inequality $L[u] \leq 0$ in $H \times G$ and satisfies the boundary condition $u=0$ on $H \times \partial G$.

Then the function $g(x)$ given by (1) satisfies the differential inequality

$$
\Delta_{x^{\prime}} g+p(x) \phi(g) \leq 0, \quad x \in H
$$

Proof. Since $\Delta_{x} g(x)=\frac{1}{k} \int_{G} \Delta_{x} u d y$, it follows from Green's formula that 


$$
\begin{aligned}
\Delta_{x} g(x) & \leq \frac{1}{\kappa} \int_{G} \sum_{i, j=1}^{m} \frac{\partial}{\partial y_{i}}\left(a_{i j}(x, y) \frac{\partial u}{\partial y_{j}}\right) d y-\frac{I}{\kappa} \int_{G} f(x, y, u) d y \\
& =\frac{1}{\kappa} \int_{\partial G} \frac{\partial u}{\partial v} d \tau-\frac{1}{\kappa} \int_{G} f(x, y, u) d y,
\end{aligned}
$$

where $\frac{\partial}{\partial v}=\sum_{i, j=1}^{m} a_{i j}(x, y) v_{i} \frac{\partial}{\partial y_{j}}, v=\left(v_{1}, \ldots, v_{m}\right)$ being the unit

exterior normal vector to $\partial G$, and $\tau$ denotes the measure on $\partial G$. In view of the fact that $u>0$ in $H \times G$ and $u=0$ on $H \times \partial G, \frac{\partial u}{\partial v}$ must be non-positive. Therefore, using hypothesis $(i)$ and Jensen's inequality applied to $\phi(u)$ over $G$, we get

$$
\begin{aligned}
\Delta_{x} g(x) & \leq-\frac{p(x)}{\kappa} \int_{G} \phi(u) d y \\
& \leq-p(x) \phi\left[\frac{I}{\kappa} \int_{G} u(x, y) d y\right],
\end{aligned}
$$

which is the desired inequality (2).

We shall use the notation

$$
H_{r}=H \cap\left\{x \in R^{n}:|x|>r\right\}, r>0 .
$$

DEFINITION. A function $u(x, y) \in D_{L}$ which satisfies

$$
u L[u] \leq 0 \text { in } H \times G \text { and } u=0 \text { on } H \times \partial G
$$

is said to be oscillatory in $H \times G$ if it has a zero in $H_{r} \times G$ for every $r>0$.

PROPOSITION 1. Every solution of (3) is oscizlatory in $H \times G$ if in addition to hypothesis ( $i$ ) of Lemma 1 the following conditions are satisfied:

(i) $f(x, y,-\xi)=-f(x, y, \xi)$ for alz $(x, y) \in H \times G$ and for all $\xi>0$;

(ii) the differential inequality (2) has no solution which is positive in ${ }_{r}$ for any $r>0$.

Proof. Suppose to the contrary that there exists a solution $u(x, y)$ 
of (3) which has no zero in $H_{r^{\prime}} \times G$ for some $r^{\prime}>0$. If $u>0$ in $H_{r^{\prime}} \times G$, then $L[u] \leq 0$ in $H_{r^{\prime}} \times G$, and by Lema 1 , the function $g(x)$ defined by $(1)$ is a positive solution of (2) in $H_{r^{\prime}}$, contradicting the hypothesis $(i i)$.

Likewise, $u$ cannot be negative in $H_{r}, G$, or else $-u$ would be a positive solution of (3).

In the case when $n=1$, the operator $L$ reduces to a hyperbolic operator and the inequality (2) becomes the ordinary differential inequality

$$
\frac{d^{2} g}{d x^{2}}+p(x) \phi(g) \leq 0, \quad x>0 .
$$

Sufficient conditions for the non-existence of eventually positive solutions of (4) have recentiy been established by Naito and Yoshida [4] and Noussair and Swanson [6]. Here we present an oscillation criterion for the semilinear hyperbolic operator $L \quad(n=1)$ which follows from Proposition 1 combined with a result of [4, Theorem 2.1].

THEOREM 1. Assume that the following conditions are satisfied:

(I) $f(x, y, \xi) \geq p(x) \phi(\xi)$ for all $(x, y) \in(0, \infty) \times G$ and for all $\xi>0$, where $p$ is continuous and non-negative in $(0, \infty)$ and $\phi$ is contiruous, non-negative, and convex in $(0, \infty)$;

(II) $f(x, y,-\xi)=-f(x, y, \xi)$ for azz $(x, y) \in(0, \infty) \times G$ and for all $\xi>0$;

(III) there exist positive continuous functions $\phi_{1}$ and $\phi_{2}$ in $(0, \infty)$ such that

(i) $\phi(\xi) \geq \phi_{1}(\xi) \phi_{2}(\xi)$ for all $\xi>0$,

(ii) $\phi_{1}$ is non-inereasing and $\phi_{2}$ is non-decreasing for alz $\xi>0$,

(iii) $\int_{\varepsilon}^{\infty} \frac{d \xi}{\phi_{2}(\xi)}<\infty$ for some $\varepsilon>0$, 


$$
\text { (iv) } \int^{\infty} \xi p(\xi) \phi_{1}(k \xi) d \xi=\infty \text { for } a \text { ll } k>0 \text {. }
$$

Then every solution of $(3) \quad(n=1)$ is oscillatory in $(0, \infty) \times G$.

COROLLARY 1. Consider the semilinear hyperbolic equation

$$
\frac{\partial^{2} u}{\partial x^{2}}-\sum_{i=1}^{m} \frac{\partial^{2} u}{\partial y_{i}^{2}}+c(x) u^{\gamma}=0,
$$

where $c(x)$ is a non-negative continuous function in $(0, \infty)$ and $\gamma>1$ is the quotient of odd integers. Every solution $u$ of (5) satisfying the boundary condition $u=0$ on $(0, \infty) \times \partial G$ is oscillatory in $(0, \infty) \times G$ if

$$
\int^{\infty} x c(x) d x=\infty
$$

Next we consider the case $n \geq 2$. Letting $(r, \theta)$ denote hyperspherical coordinates for $R^{n}, H$ can be rewritten as

$$
H=\{(r, \theta): 0<r<\infty, \theta \in \theta\},
$$

where $\theta$ is the domain defined by

$$
\theta=\left\{\theta=\left(\theta_{1}, \ldots, \theta_{n-1}\right): 0<\theta_{i}<\pi / 2, i=1, \ldots, n-1\right\} .
$$

The following notation will be used:

$$
\begin{aligned}
S_{r} & =\left\{x \in R^{n}:|x|=r\right\}, \\
H(x) & =H \cap S_{r}, \\
H(s, t) & =\{x \in H: s<|x|<t\} .
\end{aligned}
$$

The measure on $S_{p}$ and $S_{1}$ will be denoted by $\sigma$ and $\omega$, respectively. The unit exterior normal vector to $\partial H$ will be denoted by $n$. Associated with every function $u \in D_{L}$, we define a function $h(r)$ in $(0, \infty)$ by the equation

$$
h(r)=\frac{I}{\sigma_{r}} \int_{H(r)} g(x) d \sigma,
$$

where $g(x)$ is the function given by (I) and $\sigma_{p}$ denotes the area of 
$H(r)$.

By employing the technique of Noussair and Swanson [6], we obtain the following principal tool.

LEMMA 2. Assume that the hypotheses (i) and (ii) of Lemma 1 hold and, moreover, that

(i) $p(x) \geq q(|x|)$ in $H_{r_{0}}$ for some $r_{0}>0$, where $q$ is continuous and non-negative in $\left[r_{0}, \infty\right)$;

(ii) $\frac{\partial g}{\partial \eta} \geq 0$ on $\partial H_{r_{0}}$, where $g$ is given by (1).

Then the function $h(x)$ defined by (6) satisfies the ordinary differential inequaitity

$$
\frac{d}{d r}\left(r^{n-1} \frac{d h}{d r}\right)+r^{n-1} q(r) \phi(h) \leq 0, \quad r \geq r_{0} .
$$

Proof. Green's formula yields the integral identity

(8) $\int_{H\left(r_{0}, r\right)} \Delta_{x} g d x=\int_{H(r)} \frac{\partial g}{\partial r} d \sigma-r_{0}^{n-1} \int_{H(1)} \frac{\partial g}{\partial r} d \omega$

$$
+\int_{r_{0}}^{r} d \rho \int_{\partial \Theta} \frac{\partial g}{\partial \eta}(\rho, \theta) d \mu
$$

for any $r \geq r_{0}$, where $\mu$ denotes the measure on $\partial \theta$. Since the following identities hold,

$$
\begin{aligned}
\frac{d}{d r}\left(\int_{H\left(r_{0}, r\right)} \Delta_{x} g d x\right) & =\int_{H(r)} \Delta_{x} g d \sigma, \\
\frac{d}{d r}\left(\int_{H(r)} \frac{\partial g}{\partial r} d \sigma\right) & =\omega_{1} \frac{d}{d r}\left(r^{n-1} \frac{d h}{d r}\right),
\end{aligned}
$$

where $\omega_{1}$ denotes the area of $H(1)$, differentiating (8) with respect to $r$ and using condition (ii), we obtain

$$
\int_{H(r)} \Delta_{x} g d \sigma \geq \omega_{1} \frac{d}{d r}\left(r^{n-1} \frac{d h}{d r}\right) .
$$

On the other hand, applying Jensen's inequality to $\phi(g)$ over $H(r)$ and 
using condition (i), we find

$$
\omega_{1} r^{n-1} q(r) \phi(h) \leq \int_{H(r)} p \phi(g) d \sigma .
$$

The conclusion (7) now follows from Lemma $1,(9)$, and (10). This completes the proof.

PROPOSITION 2. Let the following conditions hold.

(i) $f(x, y, \xi) \geq q(|x|) \phi(\xi)$ in $H_{p_{0}} \times G \times(0, \infty)$ for some $r_{0}>0$, where $q$ is continuous and non-negative in $\left[r_{0}, \infty\right)$ and $\phi$ is continuous, non-negative, and convex in $(0, \infty)$.

(ii) $f(x, y,-\xi)=-f(x, y, \xi)$ for alz $(x, y) \in H_{r_{0}} \times G$ and for alz $\xi>0$.

(iii) The ordinary differential inequality (7) has no positive solution in $[r, \infty)$ for any $r \geq r_{0}$.

Then every solution $u$ of (3) which satisfies

$$
\frac{\partial u}{\partial \eta}-\lambda(x, y) u=0
$$

on ${ }^{\partial H_{r_{0}}} \times G$ is oscizzatory in $H \times G$, where $\lambda(x, y)$ is a non-negative continuous function on ${ }^{\partial H_{r_{0}}} \times G$.

Proof. If $u$ is a solution of (3) which satisfies (11) and is positive in $H_{r_{1}} \times G$ for some $r_{1} \geq r_{0}$, then we find from (1) and (11) that

$$
\begin{aligned}
\frac{\partial g}{\partial n} & =\frac{1}{\kappa} \int_{G} \frac{\partial u}{\partial \eta} d y \\
& =\frac{1}{\kappa} \int_{G} \lambda(x, y) u d y \geq 0 .
\end{aligned}
$$

Define the function $h(r)$ by (6). Then, proceeding as in the proof of Proposition 1 and using Lemma 2, we can show that $h(r)$ is a positive solution of (7) in $\left[r_{1}, \infty\right)$. But this is a contradiction. 
The above proposition together with the results of Naito and Yoshida [4, Theorems 2.1 and 2.4] yields the following oscillation criteria for the semilinear ultrahyperbolic operator $L$.

THEOREM 2. Let $n=2$ and assume that:

(i) the hypotheses (i) and (ii) of Proposition 2 are satisfied;

(ii) there exist positive continuous functions $\phi_{1}$ and $\phi_{2}$ in $(0, \infty)$ such that (i), (ii), and (iii) of Theorem 1 (III) hold, and

$$
\int^{\infty} \xi(\log \xi) q(\xi) \phi_{1}(k \log \xi) d \xi=\infty \text { for } \text { all } k>0 \text {. }
$$

Then every solution of (3) satisfying (11) is oscillatory in $H \times G$.

THEOREM 3. Let $n \geq 3$ and suppose that:

(i) the hypotheses (i) and (ii) of Proposition 2 are satisfied;

(ii) there exist positive continuous functions $\phi_{1}, \phi_{2}, \phi_{3}$, and $\Phi_{4}$ in $(0, \infty)$ such that

$\phi(\xi) \geq \phi_{1}(\xi) \phi_{2}(\xi)$ for all $\xi>0$,

$\phi_{1}$ is non-increasing and $\phi_{2}$ is non-decreasing for all $\xi>0$, $\phi_{2}(\xi \zeta) \geq \phi_{3}(\xi) \phi_{4}(\zeta)$ for all $\xi, \zeta$ such that $0<\xi<1 / \zeta$, $\int_{\varepsilon}^{\infty} \frac{d \xi}{\phi_{3}(\xi)}<\infty$ for some $\varepsilon>0$, $\int^{\infty} \xi^{n-1} q(\xi) \phi_{4}\left(\frac{\xi^{2-n}}{m-2}\right) d \xi=\infty$.

Then every solution of (3) satisfying (11) is oscillatory in $H \times G$.

COROLLARY 2. Consider the semilinear ultrahyperbolic equation

$$
\sum_{i=1}^{n} \frac{\partial^{2} u}{\partial x_{i}^{2}}-\sum_{j=1}^{m} \frac{\partial^{2} u}{\partial y_{j}^{2}}+c(|x|) u^{\gamma}=0,
$$

where $c$ is a non-negative continuous function in $(0, \infty)$ and $\gamma>1$ is the quotient of odd integers. Every solution $u$ of (12) satisfying (11) 
and $u=0$ on $H \times \partial G$ is oscilzatory in $H \times G$ if

$$
\int_{n}^{\infty} \psi_{n}(r) c(r) d r=\infty
$$

where

$$
\Psi_{n}(r)= \begin{cases}r \log r & \text { if } n=2, \\ p^{n-1+\gamma(2-n)} & \text { if } n \geq 3 .\end{cases}
$$

\section{References}

[1] Charles Kahane, "Oscillation theorems for solutions of hyperbolic equations", Proc. Amer. Math. Soc. 41 (1973), 183-188.

[2] Kurt Kreith, "Sturmian theorems for hyperbolic equations", Proc. Amer. Math. Soc. 22 (1969), 277-281.

[3] Kurt Kreith, "Sturmian theorems for characteristic initial value problems", Atti Accad. Naz. Lincei Rend. Cl. Sci. Fis. Mat. Natur. 47 (1969), 139-144.

[4] Manabu Naito and Norio Yoshida, "Oscillation theorems for semilinear elliptic differential operators", submitted.

[5] Mamoru Narita and Norio Yoshida, "Oscillation theorems for linear ultrahyperbolic operators", submitted.

[6] E.S. Noussair and C.A. Swanson, "Oscillation theory for semilinear Schrödinger equations and inequalities", Proc. Roy. Soc. Edinburgh Sect. A 75 (1975-1976), 67-81.

[7] Gordon Pagan, "Oscillation theorems for characteristic initial value problems for linear hyperbolic equations", Atti Accad. Naz. Lincei Rend. C2. Sci. Fis. Mat. Natur. 55 (1973), 301-313.

[8] C.C. Travis, "Comparison and oscillation theorems for hyperbolic equations", Utilitas Math. 6 (1974), 139-151. 
[9] Eutiquio C. Young, "Comparison and oscillation theorems for singular hyperbolic equations", Atti Accad. Naz. Lincei Rend. Cl. Sci. Fis. Mat. Natur. 59 (1975), 383-391.

Department of Mathematics,

Faculty of Science,

Hi roshima University,

Hi roshima,

Japan. 